\title{
From isoparametric submanifolds to polar foliations
}

\author{
Gudlaugur Thorbergsson ${ }^{1}[$
}

Accepted: 1 February 2022 / Published online: 23 February 2022

(c) The Author(s) 2022

\begin{abstract}
We will show how isoparametric submanifolds and polar actions on round spheres lead to polar foliations and polar actions on compact symmetric spaces and compact Riemannian manifolds with positive curvature. Our emphasis will be on the classification of these submanifolds and actions.
\end{abstract}

Keywords Isoparametric submanifolds $\cdot$ Polar actions $\cdot$ Polar foliations

\section{Introduction}

This paper is dedicated to Carlos Edgard Harle, who passed away in January of 2020. It precedes an English translation of his paper [19], in which the concept of an isoparametric submanifold with arbitrary codimension was first introduced. Our aim will be to sketch how this concept has been generalized to polar foliations and to review some of the most important results that have been achieved until now.

I first met Harle in the eighties at IMPA in Rio de Janeiro shortly after he had written the paper [19] and then again during visits to the University of São Paulo. I have very kind memories of my encounters with him and of the conversations we had in the cafeteria of the Mathematics Institute (IME-USP).

It was in the late eighties, when I was beginning my work on isoparametric submanifolds, that it came to my attention that his paper had been overlooked in the literature, maybe because it was written in Portuguese. Still today, it is often not referred to where it would be appropriate. Let us hope the English translation changes that.

Dedicated to the memory of Carlos Edgard Harle.

Communicated by Claudio Gorodski.

Gudlaugur Thorbergsson

gthorber@mi.uni-koeln.de

1 Mathematisches Institut, Universität zu Köln, Weyertal 86-90, 50931 Cologne, Germany 
The study of isoparametric hypersurfaces in Euclidean spaces started about a hundred years ago with papers dealing with problems in Geometric Optics. In the late thirties, Cartan began the study of isoparametric hypersurfaces in spheres, which turned out to be much more complex than the problem in Euclidean spaces; see [35] for a survey. The final step in the classification of isoparametric hypersurfaces in spheres has very recently been accomplished by Chi; see [6]. Isoparametric submanifolds will be the starting point of our discussion, which will then lead us to polar foliations in compact symmetric spaces and compact positively curved manifolds. We will mostly consider submanifolds with codimension at least two. For this reason, we will neither cover the exciting new results on isoparametric hypersurfaces in spheres [6] and noncompact symmetric spaces [10] nor those in exotic spheres [16] to mention only a few of the topics we have had to leave out.

\section{Harle's paper}

Before stating the definition of isoparametric submanifolds given by Harle in the paper [19], I will shortly review the hypersurface case.

A function $f$ on an open set $U$ in a Riemannian manifold $M$ such that both $\|\operatorname{grad} f\|^{2}$ and $\Delta f$ are constant on the level hypersurfaces of $f$ is said to be isoparametric and a regular level set of $f$ is called an isoparametric hypersurface. An isoparametric family is the collection of the regular level sets of an isoparametric function.

An equivalent way of requiring the constancy of $\|\operatorname{grad} f\|^{2}$ and $\Delta f$ on the level surfaces of $f$ is to ask that there are continuous functions $\alpha$ and $\beta$ on the image $f(U)$ such that

$$
\|\operatorname{grad} f\|^{2}=\alpha \circ f \quad \text { and } \quad \Delta f=\beta \circ f .
$$

The condition $\|\operatorname{grad} f\|^{2}=\alpha \circ f$ implies that the regular level sets are equidistant and $\Delta f=\beta \circ f$ means that they have constant mean curvature.

Now we come to Harle's definition in [19].

Definition 2.1 An isoparametric submanifold in an open set $U$ of a Riemannian manifold $M^{n+k}$ is the preimage of a regular value of a map

$$
F=\left(F_{n+1}, \ldots, F_{n+k}\right): U \rightarrow \mathbb{R}^{k}
$$

(1) such that the functions

$$
P_{i j}=\left\langle\operatorname{grad} F_{i}, \operatorname{grad} F_{j}\right\rangle \text { and } \Delta F_{i}
$$

are constant on the regular preimages of $F$

(2) and such that the distribution spanned by 


$$
\operatorname{grad} F_{n+1}, \ldots, \operatorname{grad} F_{n+k}
$$

over the regular level sets of $F$ is integrable.

The map $F$ in the definition is called an isoparametric map and an isoparametric family is the collection of the isoparametric submanifolds.

Condition (1) in the definition above is a straightforward generalization of the definition of an isoparametric hypersurface. The spaces spanned by the gradients of $F_{1}, \ldots, F_{k}$ are the normal spaces of the regular level sets of $F$. Condition (2) is of course trivial when $k=1$. When $k \geq 2$, condition (2) leads to the concept of a section of an isoparametric and a polar foliation, which is one of the central notions in the theory as we will see.

In [19], Harle calls a map satisfying only condition (1) isoparametric and says that it is normal isoparametric if it satisfies both (1) and (2). This terminology is not used today.

Later, another definition of isoparametric submanifolds in Riemannian manifolds was proposed by Heintze, Liu, and Olmos in [20]. As far as I know, there is no study of how the two definitions relate.

The following consequence of Definition 2.1 is proved in [19].

Proposition 2.2 Let $M$ be an isoparametric submanifold in a Riemannian manifold. Then

(1) the mean curvature vector field along $M$ has constant norm and

(2) the normal bundle of $M$ is flat and the leaves of the distribution of normal spaces of the isoparametric family are totally geodesic.

From now on, Harle restricts his attention to ambient spaces $M^{n+k}(c)$ with constant sectional curvature $c$ in [19].

Proposition 2.3 Let $M^{n}$ be an isoparametric submanifold in $M^{n+k}(c)$ and let $\xi$ be a parallel normal vector field along $M^{n}$.

(1) Let t be a real number. Then the map $f_{\xi}: M^{n} \rightarrow M^{n+k}(c)$ defined by setting

$$
f_{\xi}(q)=\exp t \xi(q)
$$

sends $M$ diffeomorphically into another isoparametric submanifold in the isoparametric family.

(2) The principal curvatures in direction $\xi$ (i.e., the eigenvalues of the shape operator $A_{\xi}$ in direction $\xi$ ) are constant along $M$.

(3) A generalization of Cartan's Fundamental Formula for isoparametric hypersurfaces holds for isoparametric families in $M^{n+k}(c)$. 
The conclusion in (2) about the constancy of principal curvatures does not hold true in ambient spaces with nonconstant sectional curvatures.

Cartan's Fundamental Formula will not play a role in this paper so we do not formulate it. It is formula (11) in Cartan's first paper on isoparametric hypersurfaces; see [4].

\section{Terng's contribution}

Carter and West gave a rather abstract definition of an isoparametric map between Riemannian manifolds in [5], which when specialized to a map $F: M \rightarrow \mathbb{R}^{2}$ turns out to be equivalent to Definition 2.1 in this special case. The meaning of their concept of an isoparametric map is not studied in other situations in [5] like replacing $\mathbb{R}^{2}$ by $\mathbb{R}^{k}$ and I do not know other papers that do so. They then study families of isoparametric submanifolds with codimension two first in general Riemannian manifolds and then in ambient spaces with constant sectional curvature. The most remarkable of their results is that they associate a Coxeter group (which they call a kaleidoscopic pattern) to isoparametric submanifolds with codimension two in space forms. We will explain this in connection with Terng's work below.

In [32], Terng defined isoparametric families with arbitrary codimension in ambient spaces with constant sectional curvature. The definition is the same as Definition 2.1 (except that the ambient space is less general). There is a reference to [5], but neither in [5] nor in [32] is there a reference to Harle's paper [19].

Now let us come to the definition of the Coxeter group in [5, 32]. First one proves that an isoparametric submanifold in $S^{m}$ is isoparametric in $\mathbb{R}^{m+1}$ and that a compact isoparametric submanifold in $\mathbb{R}^{m+1}$ is isoparametric in some round hypersphere in $\mathbb{R}^{m+1}$.

Let $M^{n}$ belong to an isoparametric family $\mathcal{F}$ in $S^{n+k}$ consisting of compact submanifolds and let $\Sigma$ be a $k$-dimensional great sphere that is tangent to the normal space of $M^{n}$ at one point $p$. We assume that $M^{n}$ is full in the sense that it is not contained in a great subsphere of $S^{n+k}$. It turns out that $\Sigma$ meets all submanifolds in $\mathcal{F}$ and that all such intersections are perpendicular. In fact, $\Sigma$ is locally a leaf of the distribution of normal spaces when it passes through submanifolds in $\mathcal{F}$. Let $F$ be the set of all focal points of $M$ in $S^{n+k}$ and consider the intersection

$$
F \cap \Sigma \text {. }
$$

Then it turns out that $F \cap \Sigma$ is a union over $p$ totally geodesic hyperspheres $S_{1}, \ldots, S_{p}$ in $\Sigma$ and that the reflections $R_{1}, \ldots, R_{p}$ of $\Sigma$ in these hyperspheres leave $F \cap \Sigma$ invariant, i.e., they permute the spheres $S_{1}, \ldots, S_{p}$. This implies that the reflections $R_{1}, \ldots, R_{p}$ generate a finite group $W$ of orthogonal maps, which is a Coxeter group by the basic theory of reflection groups; see e.g. [18], p. 37. This group is effective in the sense that it does not fix any subsphere of $\Sigma$ since $M^{n}$ is full in $S^{n+k}$.

A connected component of the complements of the mirrors $S_{1}, \ldots, S_{p}$

$$
\Sigma-\bigcup_{i=1}^{p} S_{i}
$$


is the interior of a spherical $k$-simplex, called a chamber, if $W$ is effective, i.e., if $M^{n}$ is full in $S^{n+k}$. It turns out that

$$
M^{n} \cap \Sigma=W(p),
$$

where $W(p)$ is the orbit of $p$ in $\Sigma$ under the Coxeter group $W$. Since $W$ acts simply transitively on the set of chambers in $\Sigma$, it turns out that $M^{n}$ meets $\Sigma$ precisely once in each chamber and that $M$ meets $\Sigma$ perpendicularly in all points in $W(p)$.

Now let $q$ be a boundary point of the chamber containing $p$ and let $\xi_{p}$ be the smallest tangent vector in $T_{p} \Sigma$ such that $\exp _{p} \xi_{p}=q$. Extend $\xi_{p}$ to a parallel normal field over $M$ that we denote by $\xi$. This is possible since the normal bundle is flat, see Proposition 2.2, and the fact that the normal bundle has trivial holonomy, which had already been proved in [32]. Now consider the map

$$
f_{\xi}: M^{n} \rightarrow S^{n+k}
$$

defined as in Proposition 2.3. It turns out that the image $f_{\xi}\left(M^{n}\right)$, which we will denote by $N$, is a smooth embedded manifold whose dimension is strictly smaller than the one of $M^{n}$. Furthermore, $N$ consists of focal points of $M^{n}$ and we have that

$$
N \cap \Sigma=W(q) \text {. }
$$

We will call $N$ a focal manifold. If we add all focal manifolds corresponding to points in the boundary of a fixed chamber in $\Sigma$ to the isoparametric submanifolds passing through its interior, we get a decomposition of $S^{n+k}$ into submanifolds. We will refer to this decomposition as an isoparametric foliation. One of the important consequences of what we have been discussing is that one isoparametric submanifold determines the whole isoparametric foliation it belongs to. Another one is that the set of focal points is the same for all isoparametric submanifold in the foliation.

An isoparametric submanifold $M^{n}$ in $S^{n+k} \subset \mathbb{R}^{n+k+1}$ is said to be irreducible if it cannot be written as a an extrinsic product of lower dimensional isoparametric submanifolds $M_{1}$ in $S^{m_{1}}$ and $M_{2}$ in $S^{m_{2}}$, i.e.,

$$
M^{n}=M_{1} \times M_{2} \subset S^{m_{1}} \times S^{m_{2}} \subset \mathbb{R}^{m_{1}+1} \times \mathbb{R}^{m_{2}+1}=\mathbb{R}^{n+k+1} .
$$

An important application of the Coxeter group is that an isoparametric submanifold $M^{n}$ in $S^{n+k}$ is irreducible if and only if $W$ is irreducible. From the theory of Coxeter groups, we know that $W$ is irreducible if and only if the corresponding CoxeterDynkin diagram is connected.

Münzner's famous result that the number of principal curvatures of an isoparametric hypersurface in a sphere must be one of the numbers $1,2,3$, 4, or 6, see [28], can now be formulated by saying that the Coxeter group of an isoparametric hypersurface in a sphere is crystallographic. A finite Coxeter group acting on $\mathbb{R}^{k+1}$ by reflections is said to be crystallographic if it leaves a lattice in $\mathbb{R}^{k+1}$ invariant. In [21], an inductive method was introduced to prove that the Coxeter groups of isoparametric submanifolds of spheres with arbitrary codimension are crystallographic. This excludes e.g. the symmetry group of the icosahedron as a Coxeter group of an isoparametric submanifold. 
Terng came up with new examples based on symmetric spaces in [32].

Example 3.1 Let $V=G / K$ be a Riemannian symmetric space and consider the $i$ sotropy representation of $K$ on $T_{p} V$ where $p=(K)$. Then the regular orbits of $K$ are isoparametric submanifolds in $T_{p} V$ and also in the round spheres centered around the origin containing them. The singular orbits are their focal manifolds in the isoparametric foliation.

There are inhomogeneous isoparametric hypersurfaces in spheres. The first examples are due to Ozeki and Takeuchi. They were later generalized and presented more systematically by Ferus, Karcher, and Münzner in [15].

There was of course the question whether there are irreducible inhomogeneous isoparametric submanifolds in spheres with codimension two or higher. The following theorem proved in [34], see also [29], answers this question in the negative. Its proofs rely strongly on Terng's structure theory for isoparametric submanifolds. The proof in [34] uses also the theory of Tits buildings and the one in [29] relies on the theory of homogeneous structures.

Theorem 3.2 Let $M^{n}$ be a compact irreducible isoparametric submanifold in $S^{n+k}$ with codimension at least two. Then there is a symmetric space G/K such that the set of orbits of the isotropy representation of $K$ in the unit sphere in $T_{(K)} G / K$ coincides with the isoparametric foliation of $M^{n}$ in $S^{n+k}$ up to an isometry between $T_{(K)} G / K$ and $\mathbb{R}^{n+k+1}$.

As we have pointed out, isoparametric hypersurfaces in spheres have been classified; see [6]. That fact together with Theorem 3.2 shows that isoparametric submanifolds have now been classified without restrictions on their codimension.

\section{Polar actions and foliations}

An isometric action of a Lie group $G$ on a Riemannian manifold $M$ is said to be polar if there is a complete isometrically immersed submanifold $\Sigma$, called a section, that meets all orbits of $G$ in such a way that all intersections between orbits and $\Sigma$ are perpendicular.

The generalized Weyl group $W$ of a polar action $G$ on $M$ is by definition

$$
W=N_{G}(\Sigma) / Z_{G}(\Sigma),
$$

where $N_{G}(\Sigma)$ is the normalizer and $Z_{G}(\Sigma)$ is the centralizer of $\Sigma$ under the action of G.

The codimension of a principal orbit of an action (polar or not) is called its cohomogeneity. All cohomogenity-one actions are polar.

Polar actions were introduced by Szenthe in [31] using a different terminology. He proved among other things that a section of a polar action is totally geodesic. His work was a continuation of that of Conlon on what we now call polar actions with 
flat sections, see [8]. The concept of a generalized Weyl group was introduced by Conlon in [8] and Szenthe in [31]. The following result of Dadok in [9] is basic for the theory of polar actions.

Theorem 4.1 Let $\rho: G \rightarrow \mathrm{O}(n)$ be an orthogonal representation of a compact Lie group that gives rise to a polar action on $\mathbb{R}^{n}$.Then there is a symmetric space $V$ whose isotropy representation has the same orbits as $G$, where we have identified $\mathbb{R}^{n}$ with the tangent space of the symmetric space.

The generalized Weyl group of the representation in Theorem 4.1 coincides with the Weyl group of the symmetric space $V$. We have seen in Example 3.1 that the symmetric space $V$ gives rise to an isoparametric foliation. Its Coxeter group coincides with the Weyl group of $V$ and hence with the generalized Weyl group of $\rho$; see [32].

Let $M$ be a smooth manifold and let $\mathcal{F}$ denote a decomposition of $M$ into smooth submanifolds, called leaves, which do not need to have the same dimension. We say that $\mathcal{F}$ is a singular foliation if

$$
T_{p} L=\left\{X_{p} \mid X \text { is a vector field tangent to the leaves of } \mathcal{F}\right\}
$$

for every leaf $L$ in $\mathcal{F}$ and every $p$ in $L$.

Now assume $M$ is a Riemannian manifold and $\mathcal{F}$ a singular foliation in $M$. Then we say that $\mathcal{F}$ is a singular Riemannian foliation if the leaves of $\mathcal{F}$ are locally equidistant.

Isoparametric foliations as defined in Sect. 3 are singular Riemannian foliations. The set of orbits of an isometric action on a Riemannian manifold is also an example of a singular Riemannian foliation, which we will refer to as an orbit foliation. The codimension of a singular foliation is the codimension of a leaf with maximal dimension.

Boualem defined polar foliations in his work [3], which was later continued by Alexandrino in [1]. A singular Riemannian foliation $\mathcal{F}$ in $M$ is said to be polar if there is for every point $p$ in $M$ a complete isometrically immersed submanifold $\Sigma$ passing through $p$, called a section, that meets all leaves of $\mathcal{F}$ in such a way that all intersections between leaves and $\Sigma$ are perpendicular.

A singular Riemannian foliation with codimension one is always polar. As in the case of actions, a section of a polar foliation is totally geodesic.

It follows from Terng's work that an isoparametric foliation is polar. Conversely, Alexandrino proved in [1] that a polar foliation in $\mathbb{R}^{n}$ and $S^{n}$ is isoparametric.

Theorem 4.2 A singular Riemannian foliation $\mathcal{F}$ in $\mathbb{R}^{n}$ or $S^{n}$ is polar if and only if it is isoparametric.

The above result of Alexandrino is a special case of a more general result in [1] where he proves that a regular leaf of a polar foliation has parallel focal structure which in Euclidean spaces and spheres is equivalent to being isoparametric. Furthermore he proves that a polar foliation restricted to a neighborhood of a point in 
a singular leaf is diffeomorphic to a piece of an isoparametric foliation. Conversely, Töben proved in [36] that a closed submanifold with a parallel focal structure in a complete Riemannian manifold defines a family of parallel and focal submanifolds that forms a polar foliation.

\section{Polar actions and foliations on higher-rank spaces}

In [33], equifocal submanifolds in compact symmetric spaces were defined. In the terminology we have been using, they can be considered to be principal leaves of polar foliations with a flat section. There is an affine Coxeter group associated to such a foliation in [33]. A polar foliation with a flat section is said to be irreducible if this affine Coxeter group is irreducible.

Christ [7] showed in the following theorem that such foliations are frequently homogeneous.

Theorem 5.1 An irreducible polar foliation with a flat section and codimension at least two in a compact symmetric space $V$ is the orbit foliation of a polar action.

It is necessary to assume that the polar foliation in Theorem 5.1 is irreducible as the example of a direct product of two inhomogeneous isoparametric hypersurfaces in spheres shows. There is a decomposition theorem for polar foliations on simply connected compact symmetric spaces in [26].

The symmetric space $V$ in Theorem 5.1 must of course have rank at least two. Kollross had already classified the polar actions with a flat section on compact irreducible symmetric spaces in his thesis which was published in [22]. In particular he classified all cohomogeneity-one actions on irreducible compact symmetric spaces. Later he showed in [23] that one can remove the condition of irreducibility of the symmetric space if the cohomogeneity of the action is at least two. A classification of cohomogeneity-one actions on reducible symmetric spaces is still unknown.

We need two definitions before we can formulate the result in [23]. We say that an isometric action of a Lie group $H$ on a Riemannian manifold $M$ is decomposable if there exist Riemannian manifolds $M_{1}$ and $M_{2}$ such that $M=M_{1} \times M_{2}$ as a Riemannian product and Lie groups $H_{1}$ acting on $M_{1}$ and $H_{2}$ on $M_{2}$ such that the action of $H_{1} \times H_{2}$ on $M=M_{1} \times M_{2}$ has the same orbits as $H$. Otherwise, we say the action is indecomposable.

Let $G$ be a compact Lie group and $H$ and $K$ two subgroups such that $(G, H)$ and $(G, K)$ are symmetric pairs. Then the action of $H$ on the compact symmetric space $G / K$ is called a Hermann action. Hermann introduced these actions to give examples of variationally complete actions. They were later found out to be polar with a flat section.

We can now formulate the result of Kollross in [23]. 
Theorem 5.2 Let $H$ be an indecomposable polar action with cohomogeneity at least two on a compact symmetric space $V$. Then $H$ has the same orbits as a Hermann action on $V$.

There were no examples of polar actions and foliations on irreducible compact symmetric spaces of rank at least two whose sections are not flat, so the question of their existence became a folklore problem.

Lytchak proved in [26] with help of Tits buildings that such polar foliations do not exist if the codimension of $\mathcal{F}$ is at least three.

Theorem 5.3 Let $M$ be a simply connected irreducible compact symmetric space with rank at least two, and let $\mathcal{F}$ be a polar foliation on $M$ with codimension at least three. Then $\mathcal{F}$ has flat sections.

The question is still not solved if the codimension of the polar foliation $\mathcal{F}$ is equal to two. Lytchak and Kollross [24] solved the problem for polar actions with cohomogeneity two, hence proving the following theorem.

Theorem 5.4 A polar action on an irreducible compact symmetric space of rank at least two has a flat section.

There is a decomposition theorem for polar actions on symmetric spaces of compact type in [25].

Many special cases of Theorem 5.4 had been proved previously, most notably under the assumption that the symmetric space is Hermitian in [2].

We have already pointed out that cohomogeneity-one actions on irreducible compact symmetric spaces where classified by Kollross in [22], but such actions on reducible spaces are still unknown. Singular Riemannian foliations with codimension one have not been investigated in compact symmetric spaces with rank at least two.

\section{Polar actions and foliations on rank-one spaces}

Polar actions on rank-one symmetric spaces. Such actions were classified in [30]. Previously, those with cohomogeneity one had be classified; see [22] and the references therein.

There are four simply connected compact rank-one symmetric spaces: the sphere $S^{n}$ and the projective spaces $P^{n}(\mathbb{C}), P^{n}(\mathbb{U})$, and $P^{2}(\mathbb{O})$.

It turns out that the sections in the projective spaces $P^{n}(\mathbb{C}), P^{n}(\mathbb{H})$, and $P^{2}(\mathbb{O})$ are totally geodesic real projective spaces $P^{k}(\mathbb{R})$. 


\subsection{Spheres}

The polar actions on $S^{n}$ are the restrictions of polar representations. These were classified by Dadok in [9]; see Theorem 4.1.

\subsubsection{Complex projective spaces}

The isotropy representation of an Hermitian symmetric space $G / K$ restricted to the unit sphere $S^{2 n+1}$ in $T_{(K)} G / K$ descends to a polar action on $P^{n}(\mathbb{C})$ and up to orbit structure all polar actions on $P^{n}(\mathbb{C})$ are of this type.

\subsubsection{Quaternionic projective spaces}

Assume $G / K$ is the product of $k$ quaternionic Kähler symmetric spaces of which at least $k-1$ have rank one. Then the action of $K$ on the unit sphere $S^{4 n+3}$ in $T_{(K)} G / K$ descends to a polar action on $P^{n}(\mathbb{H})$ and up to orbit structure all polar actions on $P^{n}(\mathbb{H})$ are of this type.

\subsubsection{Octonionic projective planes}

There is no Hopf fibration associated to $P^{2}(\mathbb{O})$. A polar action on $P^{2}(\mathbb{O})$ has cohomogeneity one or two, since these are the dimensions of the possible sections, which are totally geodesic real projective spaces. There are four examples of polar actions with cohomogeneity one and five with cohomogeneity two (one of which was overlooked in [30] and later found by Gorodski and Kollross in [17]).

\subsection{Polar foliations on rank-one symmetric spaces}

\subsubsection{Spheres}

The polar foliations in spheres coincide with the isoparametric foliations, which are classified.

\subsubsection{Complex projective spaces}

Domínguez-Vázquez determined the polar foliations in complex projective spaces with one exception in [11] as we will now explain.

A complex structure $J$ on $\mathbb{R}^{2 n+2}$ is an orthogonal, skew symmetric transformation. Let

$$
\phi_{J}: S^{2 n+1} \rightarrow P^{n}(\mathbb{C})
$$

be the corresponding Hopf map. Then a singular Riemannian foliation $\mathcal{F}$ is polar in $P^{n}(\mathbb{C})$ if and only if $\phi_{J}^{-1} \mathcal{F}$ is polar in $S^{2 n+1}$. 
Let $\mathcal{F}_{1}$ and $\mathcal{F}_{2}$ be polar foliations in $P^{n}(\mathbb{C})$ which are not congruent. Then it is possible that there are complex structures $J_{1}$ and $J_{2}$ on $\mathbb{R}^{2 n+2}$ such that $\phi_{J_{1}}^{-1} \mathcal{F}_{1}$ and $\phi_{J_{2}}^{-1} \mathcal{F}_{2}$ are congruent. An example of this was found by Xiao in [37].

Domínguez-Vázquez considered the following problem in [11]. Let $\mathcal{F}$ be an irreducible polar foliation in $S^{2 n+1}$. Find a maximal set of complex structures $J_{1}, \ldots, J_{k}$ on $\mathbb{R}^{2 n+2}$ such that $\phi_{J_{1}}(\mathcal{F}), \ldots, \phi_{J_{k}}(\mathcal{F})$ are pairwise noncongruent polar foliations in $P^{n}(\mathbb{C})$ with the same codimension as $\mathcal{F}$ in $S^{2 n+1}$.

He was able to determine such a maximal set of complex structures on $\mathbb{R}^{2 n+2}$ for all $n$ except when $n=15$ and $\mathcal{F}$ has codimension one. Notice that the last solved case in the classification of isoparametric hypersurfaces in spheres in [6] was in $S^{31}$. Still the exception in the result of Domínguez-Vázquez remains since the unsolved problem in [11] is not directly related to the one solved in [6].

One of the consequences of this classification is that every irreducible polar foliation in $P^{n}(\mathbb{C})$ is homogeneous if and only if $n+1$ is prime. Furthermore, a polar foliation $\mathcal{F}$ in $S^{2 n+1}$ gives rise to one homogeneous polar foliation in $P^{n}(\mathbb{C})$ if and only if $\mathcal{F}$ is the orbit foliation of the isotropy representation of an Hermitian symmetric space. Otherwise all of the polar foliations in $P^{n}(\mathbb{C})$ are inhomogeneous.

\subsubsection{Quaternionic projective spaces}

The polar foliations in the quaternionic projective spaces $P^{n}(\mathbb{W})$ were classified with one exception by Domínguez-Vázquez and Gorodski in [12].

We will be dealing with quaternionic structures $\mathfrak{q}$ on $\mathbb{R}^{4 n+4}$. Each such structure $\mathfrak{q}$ gives rise to a Hopf map $\phi_{\mathfrak{q}}: S^{4 n+3} \rightarrow P^{n}(\mathbb{H})$. A singular Riemannian foliation $\mathcal{F}$ in $P^{n}(\mathbb{H})$ is polar if and only if $\phi_{\mathfrak{q}}^{-1}(\mathcal{F})$ is polar in $S^{4 n+3}$.

The classification of polar foliations in $P^{n}(\mathbb{H})$ therefore leads us to the following question: Let $\mathcal{F}$ be an irreducible polar foliation in $S^{4 n+3}$. Find a maximal set of quaternionic structures $\mathfrak{q}_{1}, \ldots, \mathfrak{q}_{k}$ on $\mathbb{R}^{4 n+4}$ such that $\phi_{\mathfrak{q}_{1}}(\mathcal{F}), \ldots, \phi_{\mathfrak{q}_{k}}(\mathcal{F})$ are pairwise noncongruent polar foliations in $P^{n}(\mathbb{U})$ with the same codimension as $\mathcal{F}$ in $S^{4 n+3}$.

Domíngez-Vázquez and Gorodski solved this problem on quaternionic structures on $\mathbb{R}^{4 n+4}$ in [12] for all $n$ except $n=7$. As in the complex case, this corresponds to the last case in the classification of isoparametric hypersurfaces in spheres. Again the problem solved in [6] is not directly related to the one in [12] so that the unresolved case remains.

A consequences of the result in [12] is that an irreducible polar foliation with codimension at least two in $P^{n}(\mathbb{W})$ is homogeneous if and only if $n+1$ is prime. A polar foliation with codimension one in $P^{n}(\mathbb{H})$ is homogeneous if and only if $n$ is even or if $n=1$. There are many inhomogeneous polar foliations with higher codimension in quaternionic projective spaces.

\subsubsection{Octonionic projective spaces}

There is little known about polar foliations in the octonionic plane $P^{2}(\mathbb{O})$ except that Lytchak proved in [26] that their codimension is either one or two. 


\section{Polar actions and foliations on positively curved manifolds}

\subsection{Polar actions on positively curved manifolds}

The following theorem was proved in [14].

Theorem 6.1 A polar action of a compact Lie group $G$ on a simply connected, compact, positively curved manifold $M$ of cohomogeneity at least two is equivariantly diffeomorphic to a polar action on a compact rank-one symmetric space.

The first step in the proof is to study the possible sections of the polar action and their generalized Weyl groups; see Sect. 4. It turns out that a $k$-dimensional section $\Sigma$ is either diffeomorphic to the sphere $S^{k}$ or to the real projective space $P^{k}(\mathbb{R})$.

Theorem 6.2 The generalized Weyl group of a polar action of $G$ on a simply connected, positively curved manifold $M$ of cohomogeneity at least two is a Coxeter group or a $\mathbb{Z}_{2}$-quotient of such a group. Moreover, the section with this action is equivariantly diffeomorphic to a sphere or a real projective space with a linear action.

In the case when the section is equivariantly diffeomorphic to a sphere with a Coxeter group action and when this Coxeter group is irreducible, we can construct a Tits building and prove that $M$ is equivariantly diffeomorphic to a polar action on a round sphere.

If the section is equivariantly diffeomorphic to a real projective space we have to use more complicated methods of Tits in a proof that has many similarities with the arguments of Lytchak in [26], when proving Theorem 5.3. As there, these arguments do not work when the Coxeter group has rank three, i.e., if the cohomogeneity is equal to two. This rank-three exceptional case was dealt with in [13].

It turns out that $M$ is diffeomorphic to a rank-one symmetric space, which is not a sphere, if the section is a real projective space.

\subsection{Polar foliations on positively curved manifolds}

A preliminary study was done by Meyer in [27]. but there is still much left to be done.

\section{Summary}

We started with the definition of an isoparametric submanifold and explained some of the basic results with emphasis on spheres as ambient spaces. We then moved on to polar actions and foliations in compact symmetric spaces and explained the main results, which turned out to be rather complete. The last topic was polar actions and foliations on positively curved manifolds. 
We end with a list of the cases, which have not yet been solved.

(1) Cohomogeneity-one actions on irreducible compact symmetric spaces were classified by Kollross in [22]. Such a classification in the case of reducible compact symmetric spaces is still open.

(2) It was shown by Lytchak in [26] that polar foliations with codimension at least three on compact symmetric spaces of rank at least two have flat sections. The codimension two case is unknown except for orbit foliations. Codimension-one foliations on such spaces have not been studied.

(3) Domínguez-Vázquez and Gorodski classified polar foliations in projective spaces in $[11,12]$ with the exceptions of $P^{15}(\mathbb{C}), P^{7}(\mathbb{H})$ and $P^{2}(\mathbb{O})$ where a classification is still unknown.

(4) Much work is still to be done on polar foliations in positively curved manifolds.

Funding Open Access funding enabled and organized by Projekt DEAL.

Open Access This article is licensed under a Creative Commons Attribution 4.0 International License, which permits use, sharing, adaptation, distribution and reproduction in any medium or format, as long as you give appropriate credit to the original author(s) and the source, provide a link to the Creative Commons licence, and indicate if changes were made. The images or other third party material in this article are included in the article's Creative Commons licence, unless indicated otherwise in a credit line to the material. If material is not included in the article's Creative Commons licence and your intended use is not permitted by statutory regulation or exceeds the permitted use, you will need to obtain permission directly from the copyright holder. To view a copy of this licence, visit http://creativecommons.org/licenses/by/4.0/.

\section{References}

1. Alexandrino, M.M.: Singular Riemannian foliations with sections. Ill. J. Math. 48, 116-1182 (2004)

2. Biliotti, L.: Coisotropic and polar actions on compact irreducible Hermitian symmetric spaces. Trans. Am. Math. Soc. 358, 3003-3022 (2006)

3. Boualem, H.: Feuilletages riemanniens singuliers transversalement intégrables. Compos. Math. 95, 101-125 (1995)

4. Cartan, É.: Familles de surfaces isoparamétriques dans les espaces à courbure constante. Ann. Mat. Pura Appl. 17, 177-191 (1938)

5. Carter, S., West, A.: Isoparametric systems and transnormality. Proc. London Math. Soc. 51, 520542 (1985)

6. Chi, Q.-S.: Isoparametric hypersurfaces with four principal curvatures, IV. J. Differ. Geom. 115, 225-301 (2020)

7. Christ, U.: Homogeneity of equifocal submanifolds. J. Differ. Geom. 62, 1-15 (2002)

8. Conlon, L.: Variational completeness and K-transversal domains. J. Differ. Geom. 5, 135-147 (1971)

9. Dadok, J.: Polar coordinates induced by actions of compact Lie groups. Trans. Am. Math. Soc. 288, 125-137 (1985)

10. Díaz-Ramos, J.C., Domínguez-Vázquez, M., Sanmartín-López, V.: Submanifold geometry in symmetric spaces of noncompact type. São Paulo J. Math. Sci. 15, 75-110 (2021)

11. Domínguez-Vázquez, M.: Isoparametric foliations on complex projective spaces. Trans. Am. Math. Soc. 368, 1211-1249 (2016)

12. Domínguez-Vázquez, M., Gorodski, C.: Polar foliations on quaternionic projective spaces. Tohoku Math. J. 70(2), 353-375 (2018) 
13. Fang, F., Grove, K., Thorbergsson, G.: Rank three geometry and positive curvature. Commun. Anal. Geom. 24, 487-520 (2016)

14. Fang, F., Grove, K., Thorbergsson, G.: Tits geometry and positive curvature. Acta Math. 218, 1-53 (2017)

15. Ferus, D., Karcher, H., Münzner, H.F.: Cliffordalgebren und neue isoparametrische Hyperflächen. Math. Z. 177, 479-502 (1981)

16. Ge, J., Tang, Z.: Isoparametric functions and exotic spheres. J. Reine Angew. Math. 683, 161-180 (2013)

17. Gorodski, C., Kollross, A.: Some remarks on polar actions. Ann. Glob. Anal. Geom. 49, 43-58 (2016)

18. Grove, L.C., Benson, C.T.: Finite reflection groups. Graduate Texts in Mathematics, 2nd edn., vol. 99. Springer, New York (1985)

19. Harle, C.E.: Isoparametric families of submanifolds. (Portuguese). Bol. Soc. Brasil. Mat. 13, 35-48 (1982)

20. Heintze, E., Liu, X., Olmos, C.: Isoparametric submanifolds and a Chevalley-type restriction theorem. Integrable systems, geometry, and topology, 151-190, AMS/IP Stud. Adv. Math. 36, American Math. Soc., Providence, RI (2006)

21. Hsiang, W.-Y., Palais, R.S., Terng, C.-L.: The topology of isoparametric submanifolds. J. Differ. Geom. 27, 423-460 (1988)

22. Kollross, A.: A classification of hyperpolar and cohomogeneity one actions. Trans. Am. Math. Soc. 354, 571-612 (2002)

23. Kollross, A. A.: Hyperpolar actions on reducible symmetric spaces. Transform. Groups 22, 207-228 (2017)

24. Kollross, A., Lytchak, A.: Polar actions on symmetric spaces of higher rank. Bull. Lond. Math. Soc. 45, 341-350 (2013)

25. Kramer, L., Lytchak, A.: Homogeneous compact geometries. Transform. Groups 19, 793-852 (2014)

26. Lytchak, A.: Polar foliations of symmetric spaces. Geom. Funct. Anal. 24, 1298-1315 (2014)

27. Meyer, J.: Polar Foliations on Positively Curved Manifolds. Doctoral Dissertation. Universität Köln, (2016)

28. Münzner, H.F.: Isoparametrische Hyperflächen in Sphären. II. Über die Zerlegung der Sphäre in Ballbündel. Math. Ann. 256, 215-232 (1981)

29. Olmos, C.: Isoparametric submanifolds and their homogeneous structures. J. Differ. Geom. 38, 225234 (1993)

30. Podestà, F., Thorbergsson, G.: Polar actions on rank-one symmetric spaces. J. Differ. Geom. 53, 131-175 (1999)

31. Szenthe, J.: Orthogonally transversal submanifolds and the generalizations of the Weyl group. Period. Math. Hungar. 15, 281-299 (1984)

32. Terng, C.-L.: Isoparametric submanifolds and their Coxeter groups. J. Differ. Geom. 21, 79-107 (1985)

33. Terng, C.-L., Thorbergsson, G.: Submanifold geometry in symmetric spaces. J. Differ. Geom. 42, 665-718 (1995)

34. Thorbergsson, G.: Isoparametric foliations and their buildings. Ann. Math. (2) 133, $42-446$ (1991)

35. Thorbergsson, G.: A survey on isoparametric hypersurfaces and their generalizations. Handbook of differential geometry, Vol. I, 963-995, North-Holland, Amsterdam (2000)

36. Töben, D.: Parallel focal structure and singular Riemannian foliations. Trans. Am. Math. Soc. 358, 1677-1704 (2006)

37. Xiao, L.: Principal curvatures of isoparametric hypersurfaces in $\mathbb{C} P^{n}$. Trans. Am. Math. Soc. 352, 4487-4499 (2000)

Publisher's Note Springer Nature remains neutral with regard to jurisdictional claims in published maps and institutional affiliations. 